\title{
Preparation of value-added guava jam with addition of papaya
}

\author{
Yasar Aziz, Alam Zeb, Muhammad Uzair*, Shagufta, Muhammad Shahid \\ and Ali Muhammad \\ Department of Food Science and Technology, The University of Agriculture, Peshawar-Pakistan \\ *Corresponding author's email:uzairfst@aup.edu.pk \\ Citation \\ Yasar Aziz, Alam Zeb, Muhammad Uzair, Shagufta, Muhammad Shahid and Ali Muhammad. Preparation of value- \\ added guava jam with addition of papaya. Pure and Applied Biology. Vol. 9, Issue 1, pp554-564. \\ http://dx.doi.org/10.19045/bspab.2020.90061
}

\begin{tabular}{llll}
\hline \hline Received: 26/09/2019 & Revised: 23/11/2019 & Accepted: 03/12/2019 & Online First: 07/12/2019 \\
\hline \hline
\end{tabular}

\section{Abstract}

The aim of the study was to develop a jam rich in Vitamin $\mathrm{C}$ and antioxidants, which will act as a supplement to overcome Vitamin $C^{\text {deficiency }}$ especially in children and people having sweet tooth. Guava and Papaya were blended in jam at different ratios, i.e. 100:0 (GP $\left(\mathrm{GP}_{0}\right)$ (Guava Papaya) with no preservatives (Control), 100:0 (GP $)$, 90:10 $\left(\mathrm{GP}_{2}\right), 80: 20\left(\mathrm{GP}_{3}\right), 70: 30\left(\mathrm{GP}_{4}\right), 60: 40\left(\mathrm{GP}_{5}\right)$ and 50:50 (GP 6 ). Among these, $60 \%$ Guava pulp and 40\% Papaya pulp blended jam ( $\left.\mathrm{GP}_{5}\right)$ gave best results organoleptically. Chemical preservatives such as Potassium Metabisulphite, Potassium Sorbate and Sodium Benzoate were applied separately and in combination in various concentrations with total 6 treatments. Samples were tested for $\mathrm{pH}$, titratable acidity, total soluble solids (TSS), ascorbic acid, reducing sugar, non-reducing sugar, and sensory attributes i.e. color, flavor, texture and overall acceptability. Data was analyzed with complete randomized design (CRD). The effect of storage and treatments were studied for three months of storage with an interval of 15 days. During storage, $\mathrm{pH}$ decreased from 3.70 to 3.25 , titratable acidity increased from $0.65 \%$ to $0.84 \%$, TSS increased from 67.85 to $70.75^{\circ}$ Brix, ascorbic acid decreased from 18.35 to $8.87 \mathrm{mg} / 100 \mathrm{~g}$, reducing sugar increased from 17.49 to $29.97 \%$, non-reducing sugar decreased from 45.58 to $19.25 \%$, mean score of judges for color decreased from 7.95 to 1.45 ., flavor means felt from 8.35 to 1.45 , texture means declined from 7.75 to 1.65 and overall acceptability decreased from 8.25 to 1.55 . The results showed that the treatments and 90 days storage interval have significant effect on acceptability of Guava Papaya blended jam. The treatment $\mathrm{GP}_{5}$ and $\mathrm{GP}_{6}$ were found most acceptable. The addition of $0.05 \%$ each Sodium Benzoate and Potassium Sorbate to the jam proved to be the best combination in terms of extending the shelf life and improving the quality of Guava-Papaya blended jam during storage.

Keywords: Guava; Jam; Organoleptically; Papaya; Pectin; Physicochemical

\section{Introduction}

Guava (Psidium guajava L.) belongs to Myrtaceae family. It is an important fruit crop of sub-tropical and tropical areas of the world. It has five different species namely $P$. chinensis, $\quad P$. cattleianum, $\quad P$.
Friedrichsthalianum, Psidium guianense and $P$. guajavain which the most consumed and cultivated [1]. Pakistan ranked $2^{\text {nd }}$ after India [2] with 495.2 tones production of guava annually [3] .According to the international and local economy guava is considered a 
chief article of trade due to least agriculture requirement and its high dietary importance [4].

Guava is consumed raw or cooked because of its sweet taste. High quality jam is prepared from guava. It contains more vitamin $\mathrm{C}$ (88.2 to $250.8 \mathrm{mg} / 100 \mathrm{~g}$ ) compared to citrus, apple and ber and also contains beta carotene, phenols, pectin [5] cellulose and hemicelluloses [6].

Guava is the most appetizing and succulent fruit. Superb pudding, salad, jelly and jam, Ready to serve, squash and nectar and other value added and palatable products like cheese and canned fruit, candies and ice cream can be made from guava fruit [7]. The sensory and nutritive properties of guava make it possible for the consumers to consumed raw or make other palatable, wholesome and nourishing products like marmalades and juices [8]. Vitamin C, phenols, flavonoids and dietary fiber in guava have the capacity to fight against disorders and use as a defending tool to eliminate the oxidative stress and other diseases [9].

Papaya (Carica papaya L.) a tropical fruit belongs to the Caricaceae family, widely cultivated in tropical as well as sub-tropical regions [10]. In Australia and few West Indies' islands, it is named as pawpaw and papaw which is a wild Asiminatriloba Dunal, of Annonaceae family [11]. It has been reported by FAO in 1998 that 5.1 million metric ton papaya has been produced annually all over the world. The largest producer of papaya is Brazil followed by Mexico and Nigeria [12]. In Pakistan total area under cultivation is 1671 hectares, 1545 hectares , 1537 hectares, 1500 hectares, 1426 hectares and production was 9325 tons, 8732 tons, 7932 tons, 7708 tons, 6861 tons from 2007 to 2012 respectively [3].

Papaya has pleasant sensory characteristics especially the taste and aroma [13]. Papaya is renowned as power house of nutrients because of rich source of carotenoids,
Vitamin C, Vitamin E, niacin, riboflavin, Vitamin K, carbohydrate, folate, pantothenic acid and dietary fibers. It is also rich in Manganese, Zinc, Calcium, Copper, Sodium, Iron and Potassium [14]. The Dietary Reference Intake of Papaya $(100 \mathrm{~g})$ is $9 \%$ of Copper, 6-8\% of Magnesium and less than $3 \%$ other minerals [15].

The papaya fruit usually consumed fresh in breakfast as well as dessert fruit along with honey. The fruit in green form is used in salads and in the preparation of squash [11]. Different products like beverages, ice creams, liquors and intermediate moisture food like jam are manufactured for utilization in the food industries. The aroma of papaya and phenol containing composites are the chief characteristics and qualities which describe the papaya fruit popularity and also persuade the buyer acceptability. The papaya has been used as a food and sometime as a medicine [16]. Popularity of papaya is also to use it as a dessert or processed into jam, puree or wine [17].

Jam can be prepared from different fruits by activating the pectin in fruits by heating it with the addition of sugar and water [18]. Jam is prepared by heating the pulp of the fruits or vegetables added with water and sugar to get a desired consistency [19]. The type of fruit or vegetables and different processing conditions affects the sensory and nutritional properties of Jam [20]. Different fruit mixtures can be utilized for the preparation of jam to produce a product with good texture. Heating reduce the moisture content and sugar act as a preservative, sweetener, and gives a better consistency and texture to the end product and also helps in gel formation [21]. The biochemical properties of the jam prepared from fruit blends are different from the jam prepared from one type of fruit which can be stored by keeping good microbial, sensory and textual characters [22].

The aim of this research work is to develop a suitable combination of guava and papaya for 
the preparation of blended jam and the effect of chemical preservatives on its shelf life. In addition, the guava papaya jam would give consumer a new product with high nutritive value.

\section{Materials and methods}

This research was conducted in Food Science and Technology Laboratory, The University of Agriculture Peshawar, Khyber Pakhtunkhwa, Pakistan.

\section{Selection of fruit}

Fresh, fine and superior quality, sound papaya with most favorable maturity was picked from the local market of Karachi and guava was taken from the local market of Peshawar.

\section{Preparation of the sample}

Fruits were first sorted by hand and then graded to get the good quality. The fruits were graded on the basis of purity and firmness, size and shape, maturity and mechanical injury.The fruits were washed through running water to clean dust, sand, debris and other unwanted materials. The microbial load from the surface was reduced by dipping the fruits in hot water at a temperature of $80^{\circ} \mathrm{C}$ for the duration of two minutes.

Table 1. Treatment of Guava and papaya (GP) blended jam with different chemical preservative

\begin{tabular}{|c|c|}
\hline $\mathrm{GP}_{\mathrm{o}}$ & Control ( No preservatives) \\
\hline $\mathrm{GP}_{1}$ & $0.1 \%$ Potassium Metabisulphite \\
\hline $\mathrm{GP}_{2}$ & $0.1 \%$ Potassium Sorbate \\
\hline $\mathrm{GP}_{3}$ & $0.1 \%$ Sodium Benzoate \\
\hline $\mathrm{GP}_{4}$ & $0.05 \%$ Potassium Metabisulphite $+0.05 \%$ Potassium Sorbate \\
\hline $\mathrm{GP}_{5}$ & $0.05 \%$ Sodium Benzoate $+0.05 \%$ Potassium Sorbate \\
\hline $\mathrm{GP}_{6}$ & $0.05 \%$ Sodium Benzoate $+0.05 \%$ Potassium Metabisulphite \\
\hline
\end{tabular}

Physico-chemical, Sensory and statistical analysis

All the samples of guava papaya blended jam were subjected to evaluate physico-chemical analysis $(\mathrm{pH}$, titratable acidity, total soluble solids, reducing sugar, non-reducing sugar and ascorbic acid) by the recommended methods of [23] and sensory evaluation
The guava fruits were cut into thin slices with the help of stainless steel knife. These slices were heated in about $25 \%$ water. The slices were crushed manually after heating with the help of stainless steel sieve and a clean container was used to collect the pulp. The homogenized pulp of papaya was obtained through mixer machine. The guava and papaya pulp was mixed together in different ratios (100:0, 90:10, 80:20, 70:30, 60:40, 50:50) for the preparation of jam.

Sodium benzoate $\left(\mathrm{NaC}_{7} \mathrm{H}_{5} \mathrm{O}_{2}\right)$, Potassium Metabisulfite $\left(\mathrm{K}_{2} \mathrm{~S}_{2} \mathrm{O}_{5}\right)$ and Potassium Sorbate $\left(\mathrm{C}_{6} \mathrm{H}_{7} \mathrm{KO}_{2}\right)$ with different proportions shown in (Table 1) are used in Guava and Papaya blended jam. $\mathrm{GP}_{\mathrm{o}}$ control contains no preservatives, $\mathrm{GP}_{1}$ contains $0.1 \%$ Potassium Metabisulphite, $\mathrm{GP}_{2}$ contains $0.1 \%$ Potassium Sorbate, $\mathrm{GP}_{3}$ contains $0.1 \%$ Sodium Benzoate, $\mathrm{GP}_{4}$ contains $0.05 \%$ Potassium Metabisulphite $+0.05 \%$ Potassium Sorbate, $\mathrm{GP}_{5}$ contains $0.05 \%$ Sodium Benzoate+ $0.05 \%$ Potassium Sorbate, $\mathrm{GP}_{6}$ contains $0.05 \%$ Sodium Benzoate $+0.05 \%$ Potassium Metabisulphite The jam was stored in transparent glass jars of $250 \mathrm{ml}$ capacity at ambient temperature. 


\section{Results and discussion \\ Physico-chemical analysis \\ pH}

The mean value of $\mathrm{pH}$ was significantly $(p<0.05)$ decreased from 3.70 to 3.25 during storage. The minimum value was observed in $\mathrm{GP}_{0}$ (3.33) followed by $\mathrm{GP}_{2}$ and $\mathrm{GP}_{4}$ (3.53), while maximum value was observed in $\mathrm{GP}_{6}$ (3.62) followed by $\mathrm{GP}_{1}$ (3.61) (Table 2). The fruit juices have low $\mathrm{pH}$ because they contain higher amount of organic acids [26]. The $\mathrm{pH}$ lowered due to pectin conversion in pectic acid which was investigated by [27]. Other researchers also evaluated reduction in $\mathrm{pH}$ by studying the marmalade made by grape and apple by applying preservatives in treatments [28]. It reported decreasing trend in $\mathrm{pH}$ in all jam samples during evaluation [29].

Table 2. pH of guava-papaya blended jam during storage

\begin{tabular}{|c|c|c|c|c|c|c|c|c|}
\hline \multirow{2}{*}{ Treatments } & \multicolumn{7}{|c|}{ Storage intervals (15 Days) } & \multirow{2}{*}{ Means } \\
\cline { 2 - 9 } & Initial & $\mathbf{1 5}$ & $\mathbf{3 0}$ & $\mathbf{4 5}$ & $\mathbf{6 0}$ & $\mathbf{7 5}$ & $\mathbf{9 0}$ & \\
\hline $\mathbf{G P}_{\mathbf{0}}$ & 3.43 & 3.39 & 3.35 & 3.32 & 3.29 & 3.27 & 3.25 & 3.33e \\
\hline $\mathbf{G P}_{\mathbf{1}}$ & 3.70 & 3.67 & 3.64 & 3.61 & 3.58 & 3.56 & 3.52 & $\mathbf{3 . 6 1 a b}$ \\
\hline $\mathbf{G P}_{\mathbf{2}}$ & 3.61 & 3.58 & 3.56 & 3.53 & 3.51 & 3.48 & 3.45 & $\mathbf{3 . 5 3 d}$ \\
\hline $\mathbf{G P}_{\mathbf{3}}$ & 3.68 & 3.66 & 3.63 & 3.60 & 3.57 & 3.54 & 3.51 & $\mathbf{3 . 6 0 c}$ \\
\hline $\mathbf{G P}_{\mathbf{4}}$ & 3.62 & 3.58 & 3.56 & 3.53 & 3.50 & 3.47 & 3.45 & $\mathbf{3 . 5 3 d}$ \\
\hline $\mathbf{G P}_{\mathbf{5}}$ & 3.67 & 3.65 & 3.63 & 3.60 & 3.58 & 3.56 & 3.54 & $\mathbf{3 . 6 0 b c}$ \\
\hline $\mathbf{G P}_{\mathbf{6}}$ & 3.68 & 3.66 & 3.64 & 3.62 & 3.60 & 3.57 & 3.54 & $\mathbf{3 . 6 2 a}$ \\
\hline Means & $\mathbf{3 . 6 3 g}$ & $\mathbf{3 . 6 f}$ & $\mathbf{3 . 5 7 e}$ & $\mathbf{3 . 5 4 d}$ & $\mathbf{3 . 5 2 c}$ & $\mathbf{3 . 4 9 b}$ & $\mathbf{3 . 4 7 a}$ & \\
\hline
\end{tabular}

Mean followed by different letters are statistically different $(\mathrm{P}<0.05)$ using LSD test.

GPo (100:0, no preservatives), GP1 (100:0, with 0.1\% Potassium Metabisulphite) $\mathbf{G P}_{2}$ (90:10 with 0.1\% Potassium Sorbate) GP3 (80:20 with0.1\% Sodium Benzoate) GP4 (70:30 with 0.05\% Potassium Metabisulphite+0.05\% Potassium Sorbate) $\mathbf{G P}_{5}$ (60:40 with $0.05 \%$ Sodium Benzoate+ 0.05\% Potassium Sorbate) GP $_{6}$ (50:50 with 0.05\% Sodium Benzoate $+0.05 \%$ Potassium Metabisulphite)

\section{Titratble acidity (\%)}

The mean value of titratable acidity was significantly $(\mathrm{P}<0.05)$ increased from 0.65 to 0.84 during storage. The minimum value was observed in $\mathrm{GP}_{0}(0.73)$ followed by $\mathrm{GP}_{2}$ $(0.74)$ while maximum value was observed in $\mathrm{GP}_{5}(0.78)$ followed by $\mathrm{GP}_{4}$ and $\mathrm{GP}_{6}(0.77)$ (Table 3). Temperature and sugar content is the main reason of raising values in acidity. Breakdown of sugar contents as well as greater value of total soluble solids increased the percent acidity. It was found that acidity increased with storage time [30]. Breakdown of pectin into pectic acid resulted rise in percent acidity was also confirmed by [31].

\section{Total soluble solid (TSS) ( ${ }^{\circ}$ brix)}

The mean value of Total Soluble Solid (TSS) was significantly $(\mathrm{P}<0.05)$ increased from 67.85 to 70.75 during storage. The minimum value was observed in $\mathrm{GP}_{4}$ (68.46) followed by $\mathrm{GP}_{5}$ and $\mathrm{GP}_{6}(68.65)$, while maximum value was observed in $\mathrm{GP}_{0}$ (69.14) followed by $\mathrm{GP}_{3}$ (68.81) (Table 4). Increase in total soluble solids of guava papaya blended jam may be due to conversion of sucrose into fructose and glucose because of temperature. Study unearthed that diet jam of dried apricot shows increment in the total soluble solids values during storage [32]. Similarly other study showed similar results by using preservatives in strawberry jam [33]. 
Table 3. Titratable acidity $(\%)$ of guava-papaya blended jam during storage

\begin{tabular}{|c|c|c|c|c|c|c|c|c|}
\hline \multirow{2}{*}{ Treatments } & \multicolumn{7}{|c|}{ Storage intervals (15 Days) } & \multirow{2}{*}{ Means } \\
\cline { 2 - 9 } & Initial & $\mathbf{1 5}$ & $\mathbf{3 0}$ & $\mathbf{4 5}$ & $\mathbf{6 0}$ & $\mathbf{7 5}$ & $\mathbf{9 0}$ & \\
\hline $\mathbf{G P}_{\mathbf{0}}$ & 0.65 & 0.67 & 0.70 & 0.73 & 0.75 & 0.78 & 0.80 & $\mathbf{0 . 7 3 e}$ \\
\hline $\mathbf{G P}_{\mathbf{1}}$ & 0.67 & 0.69 & 0.72 & 0.75 & 0.78 & 0.80 & 0.83 & $\mathbf{0 . 7 5 d}$ \\
\hline $\mathbf{G P}_{\mathbf{2}}$ & 0.67 & 0.70 & 0.72 & 0.74 & 0.77 & 0.79 & 0.81 & $\mathbf{0 . 7 4 d}$ \\
\hline $\mathbf{G P}_{\mathbf{3}}$ & 0.69 & 0.72 & 0.74 & 0.76 & 0.79 & 0.81 & 0.82 & $\mathbf{0 . 7 6}$ \\
\hline $\mathbf{G P}_{\mathbf{4}}$ & 0.71 & 0.73 & 0.75 & 0.77 & 0.79 & 0.81 & 0.83 & $\mathbf{0 . 7 7 b}$ \\
\hline $\mathbf{G P}_{\mathbf{5}}$ & 0.72 & 0.74 & 0.76 & 0.79 & 0.81 & 0.83 & 0.84 & $\mathbf{0 . 7 8 a}$ \\
\hline $\mathbf{G P}_{\mathbf{6}}$ & 0.70 & 0.72 & 0.75 & 0.78 & 0.80 & 0.82 & 0.83 & $\mathbf{0 . 7 7 b}$ \\
\hline Means & $\mathbf{0 . 6 9 a}$ & $\mathbf{0 . 7 1 b}$ & $\mathbf{0 . 7 3 c}$ & $\mathbf{0 . 7 6 d}$ & $\mathbf{0 . 7 8 e}$ & $\mathbf{0 . 8 1 f}$ & $\mathbf{0 . 8 2 g}$ & \\
\hline
\end{tabular}

Mean followed by different letters are statistically different $(\mathrm{P}<0.05)$ using LSD test.

$\mathbf{G P}_{0}$ (100:0, no preservatives), GP (100:0, with 0.1\% Potassium Metabisulphite) $\mathbf{G P}_{2}$ (90:10 with 0.1\% Potassium Sorbate) GP 3 (80:20 with0.1\% Sodium Benzoate) GP4 (70:30 with 0.05\% Potassium Metabisulphite+0.05\% Potassium Sorbate) GP5 (60:40 with 0.05\% Sodium Benzoate+ 0.05\% Potassium Sorbate) GP 6 (50:50 with 0.05\% Sodium Benzoate $+0.05 \%$ Potassium Metabisulphite)

Table 4. TSS ( ${ }^{\circ}$ Brix) of guava-papaya blended jam during storage

\begin{tabular}{|c|c|c|c|c|c|c|c|c|}
\hline \multirow{2}{*}{ Treatment } & \multicolumn{7}{|c|}{ Storage intervals(15 Days) } & \multirow{2}{*}{ Means } \\
\cline { 2 - 9 } & Initial & $\mathbf{1 5}$ & $\mathbf{3 0}$ & $\mathbf{4 5}$ & $\mathbf{6 0}$ & $\mathbf{7 5}$ & $\mathbf{9 0}$ & \\
\hline GP $_{\mathbf{0}}$ & 67.95 & 68.25 & 68.55 & 68.95 & 69.45 & 70.05 & 70.75 & $\mathbf{6 9 . 1 4 a}$ \\
\hline $\mathbf{G P}_{\mathbf{1}}$ & 67.85 & 68.05 & 68.35 & 68.65 & 69.05 & 69.45 & 70.05 & $\mathbf{6 8 . 7 8 b}$ \\
\hline $\mathbf{G P}_{\mathbf{2}}$ & 67.95 & 68.05 & 68.25 & 68.55 & 68.85 & 69.25 & 69.75 & $\mathbf{6 8 . 6 6 b}$ \\
\hline $\mathbf{G P}_{\mathbf{3}}$ & 67.95 & 68.15 & 68.35 & 68.65 & 69.05 & 69.45 & 70.05 & $\mathbf{6 8 . 8 1 b}$ \\
\hline $\mathbf{G P}_{\mathbf{4}}$ & 67.75 & 67.95 & 68.15 & 68.45 & 68.65 & 68.95 & 69.35 & $\mathbf{6 8 . 4 6 c}$ \\
\hline $\mathbf{G P}_{\mathbf{5}}$ & 68.05 & 68.15 & 68.35 & 68.55 & 68.85 & 69.15 & 69.45 & $\mathbf{6 8 . 6 5 b}$ \\
\hline $\mathbf{G P}_{\mathbf{6}}$ & 67.75 & 67.95 & 68.25 & 68.55 & 68.95 & 69.35 & 69.75 & $\mathbf{6 8 . 6 5 b}$ \\
\hline Means & $\mathbf{6 7 . 8 9 g}$ & $\mathbf{6 8 . 0 8 f}$ & $\mathbf{6 8 . 3 2 e}$ & $\mathbf{6 8 . 6 2 d}$ & $\mathbf{6 8 . 9 8 c}$ & $\mathbf{6 9 . 3 8 b}$ & $\mathbf{6 9 . 8 8 a}$ & \\
\hline
\end{tabular}

Mean followed by different letters are statistically different $(\mathrm{P}<0.05)$ using LSD test.

GP 0 (100:0, no preservatives), GP1 (100:0, with 0.1\% Potassium Metabisulphite) $\mathbf{G P}_{2}$ (90:10 with 0.1\% Potassium Sorbate) GP 3 (80:20 with0.1\% Sodium Benzoate) GP4 (70:30 with 0.05\% Potassium Metabisulphite+0.05\% Potassium Sorbate) GP5 (60:40 with 0.05\% Sodium Benzoate+ 0.05\% Potassium Sorbate) GP6 (50:50 with 0.05\% Sodium Benzoate $+0.05 \%$ Potassium Metabisulphite)

\section{Ascorbic acid (mg/100g)}

The mean value of ascorbic acid was significantly $(\mathrm{P}<0.05)$ decreased from 18.35 to 8.87 during storage. The minimum value was observed in $\mathrm{GP}_{1}$ (11.91) followed by $\mathrm{GP}_{6}$ (13.31), while maximum value was observed in $\mathrm{GP}_{5}$ (15.52) followed by $\mathrm{GP}_{4}$ (14.72) (Table 5). Vitamin $\mathrm{C}$ or ascorbic acid is a water soluble vitamin since it is quick to response and ruined as the temperature raised and also affected by light during storage. Researchers reported that ascorbic acid declined when treated with chemical preservatives they also reported that these chemicals protects the alteration in vitamins which is used for many infections and disorders [34]. The findings of the similar research work were also observed slow 
decline in ascorbic acid or vitamin $\mathrm{C}$ components in strawberry jams with three month storage [33]. It is reported that treatment with chemical preservatives reduces ascorbic acid in strawberry jam during storage [35].

Table 5. Ascorbic acid (mg/100g) of guava-papaya blended jam during storage

\begin{tabular}{|c|c|c|c|c|c|c|c|c|}
\hline \multirow{2}{*}{ Treatments } & \multicolumn{7}{|c|}{ Storage intervals (15 Days) } & \multirow{2}{*}{ Means } \\
\cline { 2 - 9 } & Initial & $\mathbf{1 5}$ & $\mathbf{3 0}$ & $\mathbf{4 5}$ & $\mathbf{6 0}$ & $\mathbf{7 5}$ & $\mathbf{9 0}$ & \\
\hline $\mathbf{G P}_{\mathbf{0}}$ & 16.40 & 15.65 & 14.76 & 13.73 & 12.63 & 11.89 & 10.83 & $\mathbf{1 3 . 7 0 d}$ \\
\hline $\mathbf{G P}_{\mathbf{1}}$ & 15.29 & 13.84 & 12.82 & 11.86 & 10.88 & 9.84 & 8.87 & $\mathbf{1 1 . 9 1 f}$ \\
\hline $\mathbf{G P}_{\mathbf{2}}$ & 17.17 & 16.31 & 15.29 & 14.36 & 13.34 & 12.26 & 11.36 & $\mathbf{1 4 . 3 0 c}$ \\
\hline $\mathbf{G P}_{\mathbf{3}}$ & 16.75 & 15.76 & 14.72 & 13.63 & 12.82 & 11.72 & 10.80 & $\mathbf{1 3 . 7 4 d}$ \\
\hline $\mathbf{G P}_{\mathbf{4}}$ & 17.29 & 16.87 & 15.84 & 14.73 & 13.79 & 12.71 & 11.82 & $\mathbf{1 4 . 7 2 b}$ \\
\hline $\mathbf{G P}_{\mathbf{5}}$ & 18.35 & 17.38 & 16.29 & 15.38 & 14.75 & 13.80 & 12.72 & $\mathbf{1 5 . 5 2 a}$ \\
\hline $\mathbf{G P}_{\mathbf{6}}$ & 16.90 & 15.06 & 14.09 & 13.15 & 12.26 & 11.30 & 10.40 & $\mathbf{1 3 . 3 1 e}$ \\
\hline Means & $\mathbf{1 6 . 8 8 a}$ & $\mathbf{1 5 . 8 4 b}$ & $\mathbf{1 4 . 8 3 c}$ & $\mathbf{1 2 . 8 3 d}$ & $\mathbf{1 2 . 9 2 e}$ & $\mathbf{1 1 . 9 3 f}$ & $\mathbf{1 0 . 9 7 g}$ & \\
\hline
\end{tabular}

Mean followed by different letters are statistically different $(\mathrm{P}<0.05)$ using LSD test.

GPo (100:0, no preservatives), GP1 (100:0, with 0.1\% Potassium Metabisulphite) GP2 (90:10 with 0.1\% Potassium Sorbate) GP3 (80:20 with0.1\% Sodium Benzoate) GP4 (70:30 with $0.05 \%$ Potassium Metabisulphite+0.05\% Potassium Sorbate) GP5 (60:40 with 0.05\% Sodium Benzoate+ 0.05\% Potassium Sorbate) GP6 (50:50 with 0.05\% Sodium Benzoate $+0.05 \%$ Potassium Metabisulphite)

\section{Reducing sugar}

The mean value of reducing sugar was significantly $(\mathrm{P}<0.05)$ increased from 17.49 to 29.97 during storage. The minimum value was observed in $\mathrm{GP}_{5}$ (21.21) followed by $\mathrm{GP}_{4}$ (21.80), while maximum value was observed in $\mathrm{GP}_{1}$ (23.45) followed by $\mathrm{GP}_{0}$ (23.40) (Table 6). The change of sucrose which is non-reducing sugar to fructose and glucose (reducing sugar) due to temperature variation and high acid can be attributed to increase reducing sugar. One of the study concluded that conversion of pectin into glucose and fructose due to temperature increase the reducing sugar during storage [36]. Similar results were envisaged from other such studies where increment in reducing sugar content is observed [37] analyzing the appleolive blended jam.

\section{Non-reducing sugar}

The mean value of non-reducing sugar was significantly $(\mathrm{P}<0.05)$ decreased from 45.58 to 19.25 during storage. The minimum value was observed in $\mathrm{GP}_{0}$ (29.46) followed by $\mathrm{GP}_{1}$ (32.67), while maximum value was observed in $\mathrm{GP}_{5}$ (35.57) followed by $\mathrm{GP}_{4}$ and $\mathrm{GP}_{6}$ (35.20) (Table 7).Transformation of total sugar to reducing sugar might be the reason of reduction in non-reducing sugar and similar results were observed where nonreducing sugars decrease by using various preservatives [38]. Similarly decrease in nonreducing sugar in banana-mashroom blended jam is observed [39].

\section{Organoleptic evaluation Color}

The mean score of judges for color was decreased from 7.95 to 1.45 . The maximum mean score was recorded in $\mathrm{GP}_{5}$ (6.99) followed by $\mathrm{GP}_{6}$ (6.82), while the minimum mean score was recorded in $\mathrm{GP}_{0}$ (4.21) followed by $\mathrm{GP}_{1}$ (5.36) (Table 8). Similar score for color was also observed in orange and pineapple blended squash [40]. Another similar study also reported color degradation while studying the sensory attributes jam [41]. Vitamin C degradation and enzymatic browning is also the main cause of low color score. Other researchers also examined 
strawberry jam and observed a decreasing trend in color [42].

\section{Flavor}

The mean score of judges for flavor was decreased from 8.35 to 1.45 . The maximum mean score was recorded in $\mathrm{GP}_{5}$ (7.44) followed by $\mathrm{GP}_{6}$ (7.34), while the minimum mean score was recorded in $\mathrm{GP}_{0}$ (4.16) followed by $\mathrm{GP}_{1}$ (5.71) (Table 9). A decline in the flavor may be due to decrease in vitamin c, fluctuations in the acid or decrease in the $\mathrm{pH}$ [37]. Other researchers in the same way calculated that consequence of storage resulted reduction in flavor occurred in preservation of the mango juice [43]. The same result was calculated in another similar study during watermelon and lemon jam [44].

Table 6. Reducing sugar (\%) of guava-papaya blended jam during storage

\begin{tabular}{|c|c|c|c|c|c|c|c|c|}
\hline \multirow{2}{*}{ Treatments } & \multicolumn{7}{|c|}{ Storage intervals(15 Days) } & \multirow{2}{*}{ Means } \\
\cline { 2 - 8 } & Initial & $\mathbf{1 5}$ & $\mathbf{3 0}$ & $\mathbf{4 5}$ & $\mathbf{6 0}$ & $\mathbf{7 5}$ & $\mathbf{9 0}$ & \\
\hline $\mathbf{G P}_{\mathbf{0}}$ & 17.65 & 18.95 & 20.55 & 22.85 & 25.35 & 28.45 & 29.97 & $\mathbf{2 3 . 4 0 a}$ \\
\hline $\mathbf{G P}_{\mathbf{1}}$ & 17.66 & 19.38 & 21.32 & 23.10 & 25.32 & 27.57 & 29.81 & $\mathbf{2 3 . 4 5 a}$ \\
\hline $\mathbf{G P}_{\mathbf{2}}$ & 17.49 & 18.41 & 19.78 & 21.29 & 22.90 & 25.31 & 28.37 & $\mathbf{2 1 . 9 4 b}$ \\
\hline $\mathbf{G P}_{\mathbf{3}}$ & 17.51 & 18.64 & 20.07 & 21.30 & 23.50 & 25.43 & 27.60 & $\mathbf{2 2 . 0 1 b}$ \\
\hline $\mathbf{G P}_{\mathbf{4}}$ & 17.60 & 18.67 & 19.99 & 21.50 & 23.08 & 24.77 & 27.02 & $\mathbf{2 1 . 8 0 b c}$ \\
\hline $\mathbf{G P}_{\mathbf{5}}$ & 17.53 & 18.31 & 19.36 & 20.73 & 22.21 & 24.12 & 26.23 & $\mathbf{2 1 . 2 1 c}$ \\
\hline $\mathbf{G P}_{\mathbf{6}}$ & 17.68 & 19.39 & 21.33 & 23.11 & 25.36 & 27.07 & 29.62 & $\mathbf{2 3 . 3 7 a}$ \\
\hline Means & $\mathbf{1 7 . 5 9 g}$ & $\mathbf{1 8 . 8 2 f}$ & $\mathbf{2 0 . 3 4 e}$ & $\mathbf{2 0 . 2 7 d}$ & $\mathbf{2 3 . 9 6 c}$ & $\mathbf{2 6 . 1 0 b}$ & $\mathbf{2 8 . 3 7 a}$ & \\
\hline
\end{tabular}

Mean followed by different letters are statistically different $(\mathrm{P}<0.05)$ using LSD test.

GPo (100:0, no preservatives), GP1 (100:0, with 0.1\% Potassium Metabisulphite) GP2 (90:10 with 0.1\% Potassium Sorbate) GP3 (80:20 with0.1\% Sodium Benzoate) GP4 (70:30 with 0.05\% Potassium Metabisulphite+0.05\% Potassium Sorbate) GP5 (60:40 with 0.05\% Sodium Benzoate+ 0.05\% Potassium Sorbate) GP6 (50:50 with 0.05\% Sodium Benzoate $+0.05 \%$ Potassium Metabisulphite)

Table 7. Non-reducing sugar $(\%)$ of guava-papaya blended jam during storage

\begin{tabular}{|c|c|c|c|c|c|c|c|c|}
\hline \multirow{2}{*}{ Treatments } & \multicolumn{7}{|c|}{ Storage intervals (15 Days) } & \multirow{2}{*}{ Means } \\
\cline { 2 - 9 } & Initial & $\mathbf{1 5}$ & $\mathbf{3 0}$ & $\mathbf{4 5}$ & $\mathbf{6 0}$ & $\mathbf{7 5}$ & $\mathbf{9 0}$ & \\
\hline $\mathbf{G P}_{\mathbf{0}}$ & 40.35 & 36.23 & 33.15 & 29.90 & 25.06 & 22.29 & 19.25 & $\mathbf{2 9 . 4 6 d}$ \\
\hline $\mathbf{G P}_{\mathbf{1}}$ & 42.05 & 39.40 & 36.29 & 33.16 & 29.51 & 25.04 & 23.27 & $\mathbf{3 2 . 6 7 c}$ \\
\hline $\mathbf{G P}_{\mathbf{2}}$ & 42.23 & 39.25 & 36.16 & 32.40 & 28.35 & 25.62 & 21.38 & $\mathbf{3 2 . 2 0 a}$ \\
\hline $\mathbf{G P}_{\mathbf{3}}$ & 42.95 & 41.23 & 38.15 & 34.54 & 31.77 & 27.18 & 23.60 & $\mathbf{3 4 . 2 0 b}$ \\
\hline $\mathbf{G P}_{\mathbf{4}}$ & 45.58 & 42.29 & 39.36 & 35.23 & 31.71 & 28.18 & 24.04 & $\mathbf{3 5 . 2 0 a}$ \\
\hline $\mathbf{G P}_{\mathbf{5}}$ & 43.15 & 41.41 & 39.45 & 36.66 & 32.24 & 29.51 & 26.57 & $\mathbf{3 5 . 5 7 c}$ \\
\hline $\mathbf{G P}_{\mathbf{6}}$ & 44.05 & 41.49 & 38.71 & 35.60 & 32.16 & 28.61 & 25.75 & $\mathbf{3 5 . 2 0 a}$ \\
\hline Means & $\mathbf{4 2 . 9 1 a}$ & $\mathbf{4 0 . 1 9 b}$ & $\mathbf{3 7 . 3 2 c}$ & $\mathbf{3 3 . 9 3 d}$ & $\mathbf{3 0 . 1 1 e}$ & $\mathbf{2 6 . 6 3 f}$ & $\mathbf{2 3 . 4 1 g}$ & \\
\hline
\end{tabular}

Mean followed by different letters are statistically different $(\mathrm{P}<0.05)$ using LSD test.

GP 0 (100:0, no preservatives), GP1 (100:0, with 0.1\% Potassium Metabisulphite) GP (90:10 with 0.1\% Potassium Sorbate) $\mathbf{G P}_{3}$ (80:20 with0.1\% Sodium Benzoate) $\mathbf{G P}_{4}$ (70:30 with $0.05 \%$ Potassium Metabisulphite+0.05\% Potassium Sorbate) GP5 (60:40 with 0.05\% Sodium Benzoate+ 0.05\% Potassium Sorbate) GP6 (50:50 with 0.05\% Sodium Benzoate $+0.05 \%$ Potassium Metabisulphite) 
Table 8. Color of guava-papaya blended jam during storage

\begin{tabular}{|c|c|c|c|c|c|c|c|c|}
\hline \multirow{2}{*}{ Treatments } & \multicolumn{7}{|c|}{ Storage intervals (15 Days) } & \multirow{2}{*}{ Means } \\
\cline { 2 - 9 } & Initial & $\mathbf{1 5}$ & $\mathbf{3 0}$ & $\mathbf{4 5}$ & $\mathbf{6 0}$ & $\mathbf{7 5}$ & $\mathbf{9 0}$ & \\
\hline $\mathbf{G P}_{\mathbf{0}}$ & 6.55 & 6.05 & 5.35 & 4.45 & 3.25 & 2.35 & 1.45 & $\mathbf{4 . 2 1 d}$ \\
\hline $\mathbf{G P}_{\mathbf{1}}$ & 6.85 & 6.35 & 6.05 & 5.65 & 4.95 & 4.25 & 3.45 & $\mathbf{5 . 3 6 c}$ \\
\hline $\mathbf{G P}_{\mathbf{2}}$ & 6.95 & 6.65 & 6.35 & 5.95 & 5.45 & 4.85 & 4.15 & $\mathbf{5 . 7 6 c}$ \\
\hline $\mathbf{G P}_{\mathbf{3}}$ & 7.55 & 7.25 & 6.95 & 6.55 & 6.15 & 5.65 & 4.95 & $\mathbf{6 . 4 4 b}$ \\
\hline $\mathbf{G P}_{\mathbf{4}}$ & 7.55 & 7.25 & 6.95 & 6.65 & 6.25 & 5.95 & 5.25 & $\mathbf{6 . 5 5 a b}$ \\
\hline $\mathbf{G P}_{\mathbf{5}}$ & 7.95 & 7.65 & 7.35 & 7.05 & 6.75 & 6.35 & 5.85 & $\mathbf{6 . 9 9 a b}$ \\
\hline $\mathbf{G P}_{\mathbf{6}}$ & 7.75 & 7.45 & 7.25 & 6.95 & 6.55 & 6.15 & 5.65 & $\mathbf{6 . 8 2 a}$ \\
\hline Means & $\mathbf{7 . 3 1 f}$ & $\mathbf{6 . 9 5 e}$ & $\mathbf{6 . 6 1 d}$ & $\mathbf{6 . 1 8 c}$ & $\mathbf{5 . 6 2 b c}$ & $\mathbf{5 . 0 8 a b}$ & $\mathbf{4 . 3 9 a}$ & \\
\hline
\end{tabular}

Mean followed by different letters are statistically different $(\mathrm{P}<0.05)$ using LSD test.

GP $_{0}$ (100:0, no preservatives), GP (100:0, with 0.1\% Potassium Metabisulphite) GP $_{2}$ (90:10 with 0.1\% Potassium Sorbate) GP$_{3}$ (80:20 with $0.1 \%$ Sodium Benzoate) GP$_{4}$ (70:30 with $0.05 \%$ Potassium Metabisulphite $+0.05 \%$ Potassium Sorbate) GP5 (60:40 with 0.05\% Sodium Benzoate+ 0.05\% Potassium Sorbate) GP 6 (50:50 with 0.05\% Sodium Benzoate $+0.05 \%$ Potassium Metabisulphite)

Table 9. Flavor of guava-papaya blended jam during storage

\begin{tabular}{|c|c|c|c|c|c|c|c|c|}
\hline \multirow{2}{*}{ Treatments } & \multicolumn{7}{|c|}{ Storage intervals (15 Days) } & \multirow{2}{*}{ Means } \\
\cline { 2 - 8 } & Initial & $\mathbf{1 5}$ & $\mathbf{3 0}$ & $\mathbf{4 5}$ & $\mathbf{6 0}$ & $\mathbf{7 5}$ & $\mathbf{9 0}$ & \\
\hline $\mathbf{G P}_{\mathbf{0}}$ & 6.25 & 5.75 & 5.15 & 4.55 & 3.55 & 2.45 & 1.45 & $\mathbf{4 . 1 6 e}$ \\
\hline $\mathbf{G P}_{\mathbf{1}}$ & 6.95 & 6.65 & 6.25 & 5.85 & 5.35 & 4.75 & 4.15 & $\mathbf{5 . 7 1 d}$ \\
\hline $\mathbf{G P}_{\mathbf{2}}$ & 7.65 & 7.35 & 6.95 & 6.55 & 6.15 & 5.65 & 4.95 & $\mathbf{6 . 4 6 c}$ \\
\hline $\mathbf{G P}_{\mathbf{3}}$ & 8.15 & 7.85 & 7.45 & 6.95 & 6.45 & 6.05 & 5.35 & $\mathbf{6 . 8 9 b}$ \\
\hline $\mathbf{G P}_{\mathbf{4}}$ & 8.05 & 7.75 & 7.45 & 7.15 & 6.65 & 6.15 & 5.45 & $\mathbf{6 . 9 5 b}$ \\
\hline $\mathbf{G P}_{\mathbf{5}}$ & 8.35 & 8.05 & 7.85 & 7.55 & 7.25 & 6.75 & 6.25 & $\mathbf{7 . 4 4 a}$ \\
\hline $\mathbf{G P}_{\mathbf{6}}$ & 8.25 & 7.95 & 7.75 & 7.45 & 7.15 & 6.65 & 6.15 & $\mathbf{7 . 3 4 a}$ \\
\hline Means & $\mathbf{7 . 6 6 a}$ & $\mathbf{7 . 3 4 a b}$ & $\mathbf{6 . 9 8 b}$ & $\mathbf{6 . 5 8 c}$ & $\mathbf{6 . 0 8 d}$ & $\mathbf{5 . 4 9 e}$ & $\mathbf{4 . 8 2 f}$ & \\
\hline
\end{tabular}

Mean followed by different letters are statistically different $(\mathrm{P}<0.05)$ using LSD test.

GPo (100:0, no preservatives), GP (100:0, with 0.1\% Potassium Metabisulphite) GP$_{2}$ (90:10 with $0.1 \%$ Potassium Sorbate) GP3 (80:20 with0.1\% Sodium Benzoate) $\mathbf{G P}_{4}$ (70:30 with $0.05 \%$ Potassium Metabisulphite $+0.05 \%$ Potassium Sorbate) GP5 (60:40 with $0.05 \%$ Sodium Benzoate+ 0.05\% Potassium Sorbate) $\mathbf{G P}_{6}$ (50:50 with 0.05\% Sodium Benzoate $+0.05 \%$ Potassium Metabisulphite)

\section{Texture}

The mean score of judges for texture was decreased from7.75 to 1.65 . The maximum mean score was recorded in $\mathrm{GP}_{5}(6.89)$, followed by $\mathrm{GP}_{6}$ (6.69), while the minimum mean score was recorded in $\mathrm{GP}_{0}$ (4.69) followed by $\mathrm{GP}_{1}$ (5.54) (Table 10). Texture is the property of food products which is judged visually and by touch. The current research result is similar in terms of reduction in texture score rate with [28] in the development of grape fruit apple marmalade. Another study results also showed decline in texture of apple jam [45].

\section{Overall acceptability}

The mean score of judges for overall acceptability was decreased from 8.25 to 1.55. The maximum mean score was recorded in $\mathrm{GP}_{5}$ (7.34) followed by $\mathrm{GP}_{6}$ (7.26), while the minimum mean score was 
recorded in $\mathrm{GP}_{0}$ (4.68) followed by $\mathrm{GP}_{1}$ (5.71) (Table 11). According to a study the overall acceptability of squashes decreases with increasing days of storage period [6]. Overall qualities were affected by temperature and storage [4].

Table 10. Texture of guava-papaya blended jam during storage

\begin{tabular}{|c|c|c|c|c|c|c|c|c|}
\hline \multirow{2}{*}{ Treatments } & \multicolumn{7}{|c|}{ Storage intervals (15 Days) } & \multirow{2}{*}{ Means } \\
\cline { 2 - 9 } & Initial & $\mathbf{1 5}$ & $\mathbf{3 0}$ & $\mathbf{4 5}$ & $\mathbf{6 0}$ & $\mathbf{7 5}$ & $\mathbf{9 0}$ & \\
\hline $\mathbf{G P}_{\mathbf{0}}$ & 6.75 & 6.35 & 5.85 & 5.05 & 4.15 & 3.05 & 1.65 & $\mathbf{4 . 6 9 f}$ \\
\hline $\mathbf{G P}_{\mathbf{1}}$ & 6.85 & 6.55 & 6.15 & 5.65 & 5.15 & 4.55 & 3.85 & $\mathbf{5 . 5 4 e}$ \\
\hline $\mathbf{G P}_{\mathbf{2}}$ & 6.95 & 6.65 & 6.35 & 5.95 & 5.45 & 4.85 & 4.15 & $\mathbf{5 . 7 6 d e}$ \\
\hline $\mathbf{G P}_{\mathbf{3}}$ & 7.35 & 7.05 & 6.75 & 6.35 & 5.85 & 5.35 & 4.75 & $\mathbf{6 . 2 1 c d}$ \\
\hline $\mathbf{G P}_{\mathbf{4}}$ & 7.45 & 7.15 & 6.55 & 6.55 & 6.15 & 5.65 & 5.15 & $\mathbf{6 . 3 8 b c}$ \\
\hline $\mathbf{G P}_{\mathbf{5}}$ & 7.75 & 7.55 & 7.25 & 6.95 & 6.65 & 6.25 & 5.85 & $\mathbf{6 . 8 9 a}$ \\
\hline $\mathbf{G P}_{\mathbf{6}}$ & 7.55 & 7.35 & 7.05 & 6.75 & 6.45 & 6.05 & 5.65 & $\mathbf{6 . 6 9 a b}$ \\
\hline Means & $\mathbf{7 . 2 4 f}$ & $\mathbf{6 . 9 5 e}$ & $\mathbf{6 . 5 6 d}$ & $\mathbf{6 . 1 8 c}$ & $\mathbf{5 . 6 9 b c}$ & $\mathbf{5 . 1 1 a b}$ & $\mathbf{4 . 4 4 a}$ & \\
\hline
\end{tabular}

Mean followed by different letters are statistically different $(\mathrm{P}<0.05)$ using LSD test.

$\mathbf{G P}_{\mathbf{0}}$ (100:0, no preservatives), GP (100:0, with 0.1\% Potassium Metabisulphite) $\mathbf{G P}_{\mathbf{2}}$ (90:10 with 0.1\% Potassium Sorbate) GP 3 (80:20 with0.1\% Sodium Benzoate) $\mathbf{G P}_{4}$ (70:30 with $0.05 \%$ Potassium Metabisulphite+0.05\% Potassium Sorbate) $\mathbf{G P}_{\mathbf{5}}$ (60:40 with 0.05\% Sodium Benzoate+ 0.05\% Potassium Sorbate) GP $_{\mathbf{6}}$ (50:50 with 0.05\% Sodium Benzoate $+0.05 \%$ Potassium Metabisulphite)

Table 11. Overall acceptability of guava-papaya blended jam during storage

\begin{tabular}{|c|c|c|c|c|c|c|c|c|}
\hline \multirow{2}{*}{ Treatments } & \multicolumn{7}{|c|}{ Storage intervals (15 Days) } & \multirow{2}{*}{ Means } \\
\hline & Initial & 15 & 30 & 45 & 60 & 75 & 90 & \\
\hline & \multicolumn{7}{|c|}{ Score rate for Overall acceptability } & \\
\hline $\mathbf{G P}_{\mathbf{0}}$ & 6.75 & 6.25 & 5.75 & 5.05 & 4.25 & 3.15 & 1.55 & $4.68 \mathrm{e}$ \\
\hline $\mathbf{G P}_{1}$ & 6.95 & 6.65 & 6.35 & 5.95 & 5.35 & 4.75 & 3.95 & 5.71d \\
\hline $\mathbf{G P}_{2}$ & 7.15 & 6.85 & 6.45 & 6.05 & 5.55 & 4.95 & 4.35 & 5.91d \\
\hline $\mathbf{G P}_{3}$ & 7.75 & 7.45 & 7.15 & 6.65 & 6.15 & 5.65 & 5.15 & $6.56 \mathrm{c}$ \\
\hline $\mathbf{G P}_{4}$ & 7.95 & 7.65 & 7.35 & 6.95 & 6.55 & 6.15 & 5.55 & $6.88 \mathrm{bc}$ \\
\hline $\mathbf{G P}_{5}$ & 8.25 & 7.95 & 7.75 & 7.45 & 7.15 & 6.55 & 6.25 & 7.34a \\
\hline GP $_{6}$ & 8.15 & 7.85 & 7.65 & 7.35 & 7.05 & 6.65 & 6.15 & 7.26ab \\
\hline Means & $7.56 \mathrm{a}$ & 7.24ab & $6.92 b$ & $6.49 \mathrm{c}$ & $6.01 d$ & $5.41 \mathrm{e}$ & 4.71f & \\
\hline
\end{tabular}

Mean followed by different letters are statistically different $(\mathrm{P}<0.05)$ using LSD test.

GPo (100:0, no preservatives), GP1 (100:0, with 0.1\% Potassium Metabisulphite) GP2 (90:10) with 0.1\% Potassium Sorbate) GP (80:20 with0.1\% Sodium Benzoate) GP4 (70:30 with 0.05\% Potassium Metabisulphite+0.05\% Potassium Sorbate) GP5 (60:40 with 0.05\% Sodium Benzoate+ 0.05\% Potassium Sorbate) GP6 (50:50 with 0.05\% Sodium Benzoate $+0.05 \%$ Potassium Metabisulphite)

\section{Conclusion}

This research was conducted for the preparation of guava-papaya blended jam and preservation action of chemicals (Potassium Metabisulphite, Sodium Benzoate, Potassium Sorbate) was applied. This study demonstrated $\mathrm{GP}_{5}$ having the combination of $0.05 \%$ Sodium Benzoate and
$0.05 \%$ Potassium Sorbate showed the best results during both physicochemical and sensory evaluation as compared to other treatments.

\section{Authors' contributions}

Conceived and designed the experiments: $\mathrm{Y}$ Aziz, A Zeb \& Shagufta, Performed the experiments: Y Aziz, Supervised the experiment: 
A Zeb, Analyzed the data and helped in writing the manuscript: $M$ Uzair \& M Shahid, Contributed materials/ analysis/ tools: A Muhammad.

\section{References}

1. Hui YH (2006). Handbook of fruits and fruit processing. John Wiley \& Sons.

2. Sindh Board of Investment (2010). PreFeasibility Study Guava. Retrieved, September 14, 2019 from http://www.sbi.gos.pk/pdf/Guava.pdf.

3. Agricultural Statistics of Pakistan (201112). Government of Pakistan ministry of national food security and research (Economic Wing); Islamabad.

4. Hui YH (2004). Handbook of Fruits and Fruit Processing. Black well Publisher; Co. Iowa, (USA).

5. Kaur S, Sarkar B C, Sharma HK \& Singh C (2009). Optimization of enzymatic hydrolysis pretreatment conditions for enhanced juice recovery from guava fruit using response surface methodology. Food and Bioprocess Technol 2(1): 96-100.

6. Panda R, Panda H, Parkash K, \& Panda A (2009). Guava pulp and leather. Sci Tech Entrepreneur India 3(1): 120-130.

7. Jain PK, Jain P \& Nema PK (2011). Quality of guava and papaya fruit pulp as influenced by blending ratio and storage period. Am J of Food Technol 6(6): 507-512.

8. Osorio C, Forero DP \& Carriazo JG (2011). Characterisation and performance assessment of guava (Psidium guajava L.) microencapsulates obtained by spraydrying. Food Res Inter 44(5): 1174-1181.

9. Yoshikawa T, Toyokuni S, Yamamoto Y \& Naito Y (2000). Free radicals in chemistry. Bio and Med OICA Int; London.

10. Rivera-Pastrana DM, Yahia EM \& González-Aguilar GA (2010). Phenolic and carotenoid profiles of papaya fruit (Carica papaya L.) and their contents under low temperature storage. $J$ of the Sci of Food and Agric 90(14): 2358-2365.

11. Medina. JDLC, Gutiérrez GV \& García HS (2013). Post-harvest Operation.

12. Benassi AC \& Cattaneo LF (2010). Report on papaya production. Work.

13. Fuggate $\mathrm{P}$, Wongs-Aree $\mathrm{C}$, Noichinda $\mathrm{S} \&$ Kanlayanarat S (2010). Quality and volatile attributes of attached and detached 'Pluk Mai Lie'papaya during fruit ripening. $S c i$ Hort 126(2): 120-129.

14. Aravind G, Bhowmik D, Duraivel S \& Harish G (2013). Traditional and medicinal uses of Carica papaya. $J$ of Med Plants Study 1(1): 7-15.

15. Wall MM (2006). Ascorbic acid, vitamin A, and mineral composition of banana (Musa sp.) and papaya (Carica papaya) cultivars grown in Hawaii. $J$ of Food Compos and anal 19(5): 434-445.

16. Medina JDLC, Gutiérrez GV \& García HS (2013). Post-harvest Operation.

17. Saeed F, Arshad MU, Pasha I, Naz R, Batool R, Khan AA \& Shafique B (2014). Nutritional and phyto-therapeutic potential of papaya (Carica papaya L.) an overview. Inter J of food Prop 17(7): 16371653.

18. Matsuura FCAU, Folegatti MIDS, Cardoso RL \& Ferreira DC (2004). Sensory acceptance of mixed nectar of papaya, passion fruit and acerola. Scientia Agricola 61(6): 604-608.

19. Usman MA \& James S (2009). Extraction and evaluation of pectin mixtures on the quality attributes of jam. Nigerian J Tropic Agric 11: 364-368.

20. Ihekoronye AI (1999). Manual on smallscale food Processing. $1^{\text {st }}$ Ed. Fijac Acad Press; Nsukka (Nigeria).

21. Battey AS, Duffy S \& Schaffner DW (2002). Modeling yeast spoilage in coldfilled ready-to-drink beverages with Saccharomyces cerevisiae, Zygosaccharomyces bailii and Candida lipolytica. Appl Environ Microbiol 68(4): 1901-1906.

22. Singh S, Jain S, Singh SP \& Singh D (2009). Quality changes in fruit jams from combinations of different fruit pulps. $J$ of Food Proc and Preser 33: 41-57.

23. AOAC (2012). Association of Official Analytical Chemists. 19 ${ }^{\text {th }}$ Ed. Arlington USA.

24. Larmond E (1977). Laboratory methods for sensory evaluation of food. Res Branch Canada Dept of Agric 128(4): 27-37. 
25. Steel RG \& Torrie JH (1997). Principles and procedures of statistics. A biochem approach $2^{\text {nd }}$ Ed. New York, pp 633.

26. Tasnim F, Hossain MA, Nusrath S, Hossain MK, Lopa D \& Haque KM (2010). Quality assessment of industrially processed fruit juices available in Dhaka city, Bangladesh. Malaysian J of Nutr 16(3): 431-438.

27. Imran A, Rafiullah K \& Muhammad A (2000). Effect of added sugar at various concentration on the storage stability of guava pulp. Sarhad J of Agric 16(1): 89-93.

28. Ehsan EB, Naeem ZP, Javed A \& Nazir A (2003). Development, standardization and storage studies on grape fruit apple marmalade. Pak J Food Sci 13(3-4): 11-15.

29. Ayub M, Ullah J, Muhammad A \& Zeb A (2010). Evaluation of strawberry juice preserved with chemical preservatives at refrigeration temperature. Inter $J$ of Nutri and Meta 2(2): 027-032.

30. Palaniswamy RP, Mukhurishman CR \& Shamuganelw KG (1974). Studies on the evaluation of certain mango verities of tainel Nadvi for pulp and squash. Indian Food Packers 28: 509.

31. Anjum FM, Din M, Ahmad I \& Pash A (2000). Preparation and evaluation of dried apricot diet jam. Pak J Food Sci 3(10): 2124.

32. Clydesdale FM, Lin YD \& Francis FJ (1972). Formation of 2-pyrrolidone-5carboxylic acid from glutamine during processing and storage of spinach puree. $J$ of Food Sci 37(1): 45-47.

33. Riaz MN, Mohyuddin G \& Al-Haq MI (1999). Physical chemical and sensory characteristics of jams made from fresh and frozen strawberries. Pak $J$ of Arid Agric (Pakistan).

34. Iqbal K, Khan A \& Khattak MMAK (2004). Biological significance of ascorbic acid (vitamin C) in human health-a review. Pak J of Nutr 3(1): 5-13.

35. Tiwari BK, O'donnell CP, Patras A, Brunton N \& Cullen PJ (2009). Effect of ozone processing on anthocyanins and ascorbic acid degradation of strawberry juice. Food Chem 113(4): 1119-1126.

36. Sawant AA \& Patil MM (2013). Preparation of guava jam blended with sapota. Agric Eng Inter CIGR J 15(1): 167-172.

37. Shah W, Khan A, Zeb A, Khan MA, Shah FN \& Amin NU (2015). Quality evaluation and preparation of apple and olive fruit blended jam. Global J of Med Res 15(1): 1421

38. Shakir I, Durrani Y, Hussainm I, Qazi IM \& Zeb A (2007). Physicochemical analysis of apple and pear mixed fruit jam prepared from varieties grown in Azad Jammu and Kashmir. Inter J of Food Safety 9: 22-24.

39. Ahmed I, Khan MU, Qazi IM, Ullah SU, Khan A \& Jamal S (2017). Development and quality evaluation of banana mushroom blended jam. Bio Sci PJSIR 60(1): 11-18.

40. Akusu OM, Kiin-Kabari DB \& Ebere CO (2016). Quality characteristics of orange/pineapple fruit juice blends. Amer $J$ of Food Sci and Techno 4(2): 43-47.

41. Gimenez J, Kajda P, Margomenou L, Piggott JR \& Zabetaki I (2001). A study on the colour and sensory attributes of highhydrostatic-pressure jams as compared with traditional jams. $J$ of the Sci of Food and Agric 81(13): 1228-1234.

42. Khan RU, Afridi SR, Ilyas M, Sohail M \& Abid H (2012). Development of strawberry jam and its quality evaluation during storage. Pak $J$ of Biochem and $\mathrm{Mol}$ Biol 45(1): 23-25.

43. Hashmi MS, Alam S, Riaz A \& Shah AS (2007). Studies on microbial and sensory quality of mango pulp storage with chemical preservatives. Pak J of Nutri 6(1): 85-88.

44. Bajwa EE, Parwaz NZ, Ghafoor \& Ghafoor (2002). Development, standardization and storage studies on watermelon lemon jam. Pak J Food Sci 12 (3-4): 21-24.

45. Muhamma A, Durrani Y, Ayu M, Zeb A \& Ullah J (2009). Organoleptic evaluation of diet apple jam from apple grown in Swat valley. Sarhad J of Agric 25(1): 81-86. 